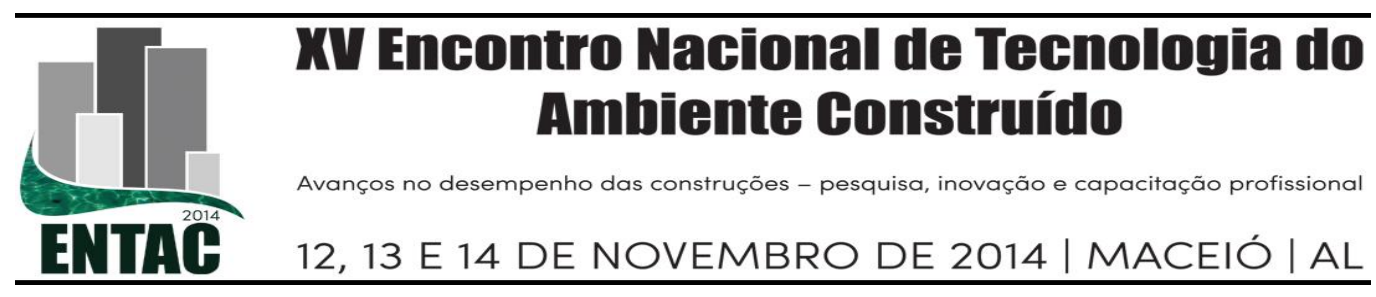

\title{
ANÁLISE COMPARATIVA QUALITATIVA (QCA) EM CANTEIROS DE OBRAS DE HABITAÇÃO DE INTERESSE SOCIAL (HIS)
}

\author{
FRANCO, Aydil de Jesus (1); PLECH, Carmem Teresa Carvalhal Cabral (3); \\ CARVALHO, Ricardo Fernandes (3)
}

(1) MEAU-UFBA, e-mail: aydil.franco@gmail.com (2) MEAU-UFBA, e-mail: carmem.apeng@gmail.com (3) MEAU-UFBA, e-mail: ricardoc@ufba.br

\begin{abstract}
RESUMO
A Construção Civil ainda é responsável por índices elevados de acidentes do trabalho e doenças profissionais. Com isso, a temática da segurança do trabalho precisa ser investigada por meio da contribuição de múltiplas áreas do conhecimento. Para tanto, este artigo tem como objetivo avaliar técnica de análise de relações lógicas, Análise Quantitativa Qualitativa, como instrumento para estabelecer relações entre variáveis qualitativas em ambiente complexo como da construção civil. Esta pesquisa faz parte do Projeto de Tecnologias para Canteiro de Obras Sustentável em Habitações de Interesse Social - CANTECHIS, que visa o desenvolvimento de soluções a serem aplicadas em canteiros de obras HIS, a fim de implementar a sustentabilidade ambiental e a melhoria das condições de trabalho. A pesquisa foi realizada em duas etapas; Na primeira, realizou-se o levantamento de dados através da aplicação de uma lista de verificação baseada na NR 18, cuja amostra foi composta por onze canteiros de obras na Região Metropolitana de Salvador. Ainda nesta etapa fez-se a tabulação dos dados dos equipamentos de proteção coletiva observados nos canteiros. A segunda, foi marcada pela comparação dessas informações com a ajuda do $f_{S} Q C A$, buscando verificar se existe uma causalidade entre as variáveis, o desempenho da segurança do trabalho e as certificações verificadas em canteiros. Contudo, e através dos resultados obtidos, espera-se a constatação de que esta técnica qualitativa possa vir a ser utilizada também nas ciências exatas, perfazendo uma ponte entre o método de pesquisa qualitativo e o quantitativo.
\end{abstract}

Palavras-chave: Habitação de Interesse Social, Análise Comparativa Qualitativa, Segurança do Trabalho

\begin{abstract}
The Construction is still responsible for high rates of occupational accidents and occupational diseases. Thus, the issue of workplace safety needs to be investigated through the contribution of multiple areas of knowledge. Therefore, this article aims to evaluate technical analysis of logical relationships, Quantitative Qualitative Analysis as a tool to establish relationships between qualitative variables in complex environment like construction. This research is part of Project Construction Technologies for Sustainable Construction in social housing - CANTECHIS, which aims the development of solutions to be applied in construction sites HIS in order to implement environmental sustainability and the improvement of working conditions. The survey was conducted in two stages; at first, we carried out the data collection by applying a checklist based on NR 18, whose sample was composed of eleven construction sites in the Metropolitan Region of Salvador. Also on stage was done tabulating the data of collective protection equipment observed in the beds. The second, was marked by comparing this information with the help of fsQCA seeking to verify if there is a causality between variables, the performance of safety and certifications verified in beds. However, and by the results obtained, we expect the qualitative finding that this technique might be also used in the hard sciences, making a bridge between the method of qualitative and quantitative research.
\end{abstract}

Keywords: Social Housing, Qualitative Comparative Analysis, Occupational Safety 


\section{INTRODUÇÃO}

O setor da construção é conhecido como uma das principais causadoras de acidentes do trabalho. Segundo Almeida et al., (2006), está em defasagem gerencial com relação a outros setores da indústria nacional devido à falta de cultura que privilegie a inovação; dificuldade em compatibilizar modelos de gestão já consagrados, dificuldades financeiras e culturais.

Com isso, estudos tem sido conduzido buscando desenvolver a cultura de segurança como um meio de desenvolver novas práticas laborais, levando a uma mudança da cultura organizacional. Segundo Glendon e Stanton (2010) a cultura organizacional é amplamente reconhecida por ser crítica para o sucesso ou o fracasso da organização demonstrando forte relação entre cultura e desempenho organizacional.

A cultura organizacional nos canteiros de obras pode ser mudada por meio de modificações na gestão dos processos, através de um conjunto de procedimentos e diretivas reunidas nas certificações de, Gestão da Qualidade (ISO 9000) que define como sendo um composto de atividades coordenadas para dirigir e controlar uma organização com relação à qualidade com foco na satisfação do cliente; Gestão Ambiental (ISO 14001) que contém regras internacionais para administração voltada à diminuição do impacto ambiental, através da definição de responsabilidades, avaliação contínua de procedimentos e avaliação contínua de processos; e, a Gestão de Segurança e Saúde (OHSAS 18001) que apesar de não ser uma norma, é um passo rumo à padronização dos Sistemas de Gestão de Segurança e Saúde no Trabalho - SGSST com foco no ambiente de trabalho seguro e saudável (FRANÇA, 2009).

Segundo Flinn (2000) tem havido a necessidade de monitorar as causas dos acidentes e incidentes, sendo medidos através de auditorias de segurança ou mensuração do clima organizacional da segurança do trabalho, através da aplicação de questionários ou listas de verificação dentro da organização ou partes dela.

Pode-se argumentar que o estudo do impacto e das relações entre fatores qualitativos, como a declaração de certificações em canteiros e o desempenho em segurança trabalho tem sido um desafio aos pesquisadores. As técnicas quantitativas da estatística impõem restrições quanto à extensão das amostras, situações controladas ou ao menos situações comparáveis, entre outras. As técnicas qualitativas são, em geral, limitadas quanto à generalização de possíveis conclusões. $O$ presente trabalho pretende avaliar técnica de análise de relações lógicas, Análise Quantitativa Qualitativa, como instrumento para estabelecer relações entre variáveis qualitativas em ambiente complexo como da construção civil.

\section{ANÁLISE COMPARATIVA QUALITATIVA (QCA)}

O QCA é uma técnica para estabelecer relações lógicas entre diferentes conceitos desenvolvida por Charles Ragin, para resolver problemas provocados pela necessidade de se fazer inferências causais com base em um pequeno número amostral de casos, buscando solucionar casos complexos analisados pelo aspecto quantitativo e qualitativo. O método tem sido utilizado nas Ciências Sociais com base na lógica binária da Álgebra Booleana e tenta maximizar o número de comparações que podem ser realizadas por meio dos casos sob investigação (RAGIN, 1987:86). O QCA possui três técnicas de análise: o Crisp-set (csQCA), o Multi-value (mvQCA) e o Fuzzy Sets $\left(f_{s} / Q C A\right)$, que trabalham, respectivamente com, álgebra booleana $(0,1)$, escala intervalar $(1,2,3,4)$ e escala contínua.

O QCA considera a causalidade conjuntural múltipla, ou seja, é possível existir mais de um resultado; sendo assim, a causalidade conjuntural múltipla contém a noção de equifinalidade, o que significa que várias combinações diferentes podem produzir o 
mesmo resultado (RAGIN, 2009). Por último, o QCA pode ainda ser usado de uma forma mais fundamentada no processo de desenvolvimento de novos argumentos teóricos, em forma de hipóteses. Com isso, para proceder com as análises, é necessária a utilização de uma das técnicas do QCA; nesta pesquisa será utilizado o $f_{s} / Q C A$.

\subsection{FUZZY-SET ANALYSIS COMO UMA ESTRATÉGIA DE ANÁLISE}

O método Fuzzy-set Analysis ( $f s / Q C A$ ) detém os aspectos chaves do QCA, permitindo a análise de fenômenos que variam de acordo com o nível ou grau, através da análise de variáveis denominadas Fuzzy. Segundo Ragin (2009:88), conjuntos fuzzy apresenta uma abordagem de dois níveis, sendo qualitativa em sua essência, mas, utilizando também métodos quantitativos. No lugar de trabalhar com variáveis binárias $(1,0)$, os conjuntos fuzzy permitem inserir valores intermediários entre ' 0 ' e ' 1 ', permitindo a percepção aproximada da complexidade das situações estudadas (RAGIN, 2009:89). Mendel e Korjani (2012) esquematizaram a sumarização do $f s / Q C A$ apresentada na Figura 1 e descrita abaixo.

\section{Figura 1 - Sumarização do $f s / Q C A$}

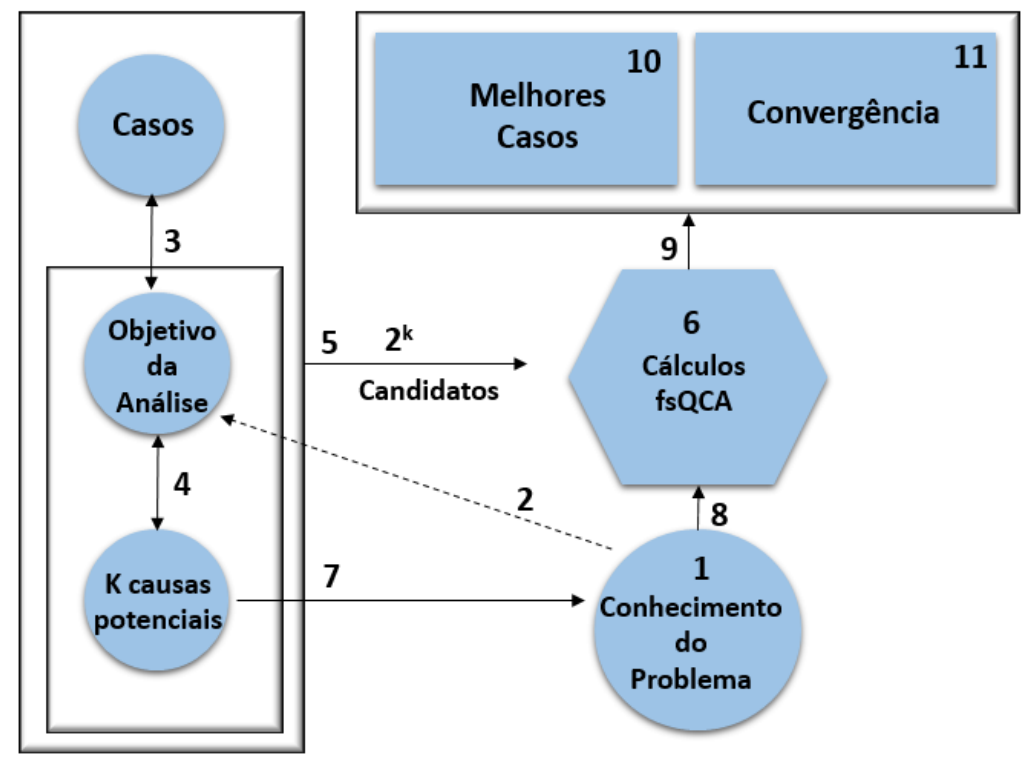

Fonte: Mendel e Korjani (2012)

A análise do $f_{s} / Q C A$ inicia-se com o estudo do problema (1) e a identificação do objetivo da análise (2). Em seguida, faz-se a escolha dos casos (3), a partir do qual se podem extrair novos conhecimentos sobre as causas possíveis para o objetivo da análise. Em seguida, postula-se um conjunto de $\mathrm{k}$ causas potenciais (4) que acredita-se ser, individualmente ou várias combinações, levando ao objetivo da análise.

$\mathrm{O}$ método $f_{s} / Q C A$ relaciona as possíveis combinações causais $2^{\mathrm{n}}$, onde $\mathrm{n}$ referese ao número de condições a serem avaliadas (5). Os dados do estudo são reduzidos a um número mínimo, e simplificam as regras, eles geralmente contêm combinações causais com menos de $\mathrm{n}$ termos (6). Este último acontece porque toda regra é para o mesmo objetivo da análise, contudo podem ser combinados logicamente usando técnicas de redução de teoria dos conjuntos, e fazendo isso, frequentemente, pode acontecer de algumas ou muitas serem condições causais absorvidos, ou seja, desaparecendo a causalidade final. Há possibilidade de não haver casos suficientes para 
fornecer combinações causais necessárias para satisfazer o objetivo da análise, entretanto, a partir do embasamento teórico é possível obter-se a condição causal (7). Este conhecimento substantivo adicional é então incorporado em outros cálculos $f_{S} / Q C A$ (8).

No final têm-se as expressões booleanas (9) que fornecem pelo menos uma combinação causal simplificada para um objetivo da análise (a menos que nenhuma regra não possa ser encontrada). Em seguida, é possível conectar casos para cada regra que são os melhores exemplos (10) e obter a convergência (11).

\section{METODOLOGIA DA PESQUISA}

A presente pesquisa envolve a realização das seguintes etapas: (a) levantamento de dados conforme metodologia adotada para o protocolo da pesquisa em rede construída pelo Projeto CANTECHIS. (b) tabulação dos dados, e (c) análise dos dados com suporte do $f_{s} / Q C A$. O levantamento dos dados foi realizado em onze canteiros de obra de Habitação de Interesse Social (HIS), do programa "Minha Casa, Minha Vida" (MCMV) na Região Metropolitana de Salvador-BA, através da aplicação de uma lista de verificação (Figura 2) com base na NR 18 (Condições e Meio Ambiente de Trabalho na Indústria da Construção) nos períodos de 2012 e 2013, sendo os canteiros envolvidos com perfil semelhante da fase de construção e número de pavimentos.

\section{Figura 2 - Trecho da lista de verificação aplicada nos canteiros de obras}

\begin{tabular}{|c|c|c|c|c|c|c|}
\hline \multicolumn{7}{|l|}{ Andaimes simplesmente apoiados } \\
\hline Os montantes dos andaimes são apoiados em sapatas sobre base sólida e resistentes? & 1 & 2 & 3 & 4 & 5 & $\mathrm{~N} / \mathrm{A}$ \\
\hline O piso de trabalho dos andaimes tem forração completa, antiderrapante, é nivelado e fixado? & 1 & 2 & 3 & 4 & 5 & N/A \\
\hline Os andaimes dispõem de guarda-corpo e rodapé? (com exceção do lado da face de trabalho) & 1 & 2 & 3 & 4 & 5 & N/A \\
\hline A proteção contra quedas por meio de guarda-corpo e rodapé : & 1 & 2 & 3 & 4 & 5 & N/A \\
\hline a) é construída com altura de $1,20 \mathrm{~m}$ para o travessão superior e $0,70 \mathrm{~m}$ para o travessão intermediário? & 1 & 2 & 3 & 4 & 5 & N/A \\
\hline hitam randanń anm alturn dn & 1 & 2 & 3 & A & 5 & $\mathrm{M} / \mathrm{\Lambda}$ \\
\hline
\end{tabular}

Vinte e quatro itens foram avaliados com base na NR 18 e, dentre esses, foi feita a redução do número de itens conforme a quantidade de equipamentos nos canteiros de obras. Para obter um resultado significativo foi considerado os equipamentos presentes em, no mínimo, três canteiros de obras. Com isso, foram selecionados os seguintes equipamentos: (1) Andaimes Simplesmente Apoiados; (2) Andaimes Fachadeiros; (3) Andaimes Suspenso Mecânico; (4) Serra Circular; (5) Máquinas, Equipamentos e Ferramentas diversas; (6) Proteção contra Choques Elétricos; (7) Escada de Mão e (8) Plataforma de Proteção.

Como os itens avaliados foram selecionados a partir da NR 18, os critérios de ponderação entre os diferentes itens avaliados foram obtidos das penalidades constantes na NR 28 (Fiscalização e Penalidades). As penalidades variam com pesos de 1 a 4, de acordo com as infrações cometidas pelas empresas. Cabe salientar que alguns itens referentes à Andaime Simplesmente Apoiados, Fachadeiros e Suspenso Mecânico, Proteção contra Choques elétricos e Escada de Mão não possuía penalidades na NR 28, porque esses itens foram retirados, respectivamente, da RTP 01 (Medidas de Proteção Contra Quedas de Altura), RTP 04 (Escadas, Rampas e Passarela) e RTP 05 (Instalações elétricas temporárias em canteiros de obras), sendo atribuído peso 0, conforme demonstra exemplo na Quadro 1. 
Quadro 1 - Fragmento da Lista de Verificação com as respectivas penalidades relacionadas

\begin{tabular}{|c|c|l|l|l|l|l|}
\hline REF & Item & \multicolumn{1}{|c|}{ Andaimes simplesmente apoiados } & Peso & \multicolumn{2}{|c|}{ Canteiros } \\
\hline NR18 & 18.15 .10 & $\begin{array}{l}\text { Os montantes dos andaimes são apoiados em sapatas sobre } \\
\text { base sólida e resistentes? }\end{array}$ & 4 & 4 & 1 & 1 \\
\hline NR18 & 18.15 .3 & $\begin{array}{l}\text { O piso de trabalho dos andaimes tem forração completa, } \\
\text { antiderrapante, é nivelado e fixado? }\end{array}$ & 4 & 0 & 2 & 2 \\
\hline NR18 & 18.15 .6 & $\begin{array}{l}\text { Os andaimes dispõem de guarda-corpo e rodapé? (Com } \\
\text { exceção do lado da face de trabalho) }\end{array}$ & 4 & 0 & 1 & 1 \\
\hline NR18 & 18.13 .5 a & $\begin{array}{l}\text { a) é construída com altura de 1,20m para o travessão superior e } \\
\text { 0,70m para o travessão intermediário? }\end{array}$ & 3 & 0 & 1 & 1 \\
\hline NR18 & 18.13 .5 b & b) tem rodapé com altura de 0,20m? & 3 & 0 & 1 & 1 \\
\hline NR18 & 18.13 .5 c & $\begin{array}{l}\text { c) tem vãos entre travessas preenchidos com tela ou outro } \\
\text { dispositivo que garanta o fechamento seguro da abertura? }\end{array}$ & 3 & 0 & 1 & 1 \\
\hline RTP 01 & pág. 10 & Os montantes distam, no máximo, 1,5 metros uns aos outros? & 0 & 0 & 1 & 1 \\
\hline
\end{tabular}

Fonte: Os Autores

Os valores a serem lançados no $f_{S} / Q C A$ foram obtidos através do cálculo da média ponderada de cada canteiro avaliado através da equação (1). Com as médias calculadas, a análise comparativa foi feita através do software $f s / Q C A$.

$$
\mathrm{MP}=\sum \frac{\text { Peso } * \text { nota }}{\text { Peso Max }}
$$

Onde, $\mathrm{MP}=$ Média ponderada;

Peso $\quad=$ Peso do item da NR 18 constantes na NR 28 (1-4);

Nota = Pontuação obtida no item avaliado (0-5);

Peso Max = Peso máximo do item avaliado conforme NR 28.

A ponderação adotada poderia corresponder a outros critérios, conforme decisão da política da empresa. Os critérios adotados correspondem à minimização de risco de multas e penalidades aos canteiros.

\section{RESULTADOS E DISCUSSÕES}

No Quadro 2 está apresentada a relação das certificações informada pelos engenheiros durante as visitas técnicas. A certificação PBQP-h (Programa Brasileiro da Qualidade e Produtividade do Habitat) não foi analisada por ser pré-requisito para as empresas construtoras que buscam linhas de financiamentos junto à Caixa Econômica Federal, inclusive aquelas que participam do programa "Minha Casa, Minha Vida" (MCMV) do Governo Federal.

Após a caracterização dos canteiros e seus respectivos equipamentos e o cálculo da média ponderada, é lançado os dados obtidos no $f_{s} / Q C A$. Cabe salientar que, segundo Ragin (2009), uma combinação é totalmente 'dentro' quando sua consistência ${ }^{1}$ é $\geq 0,80$. Nos casos avaliados, os itens Andaime Simplesmente Apoiado, Escada de Mão e

${ }^{1}$ É o grau em que as combinações têm consistência com o resultado, ou seja, quanto o total das combinações presentes na expressão explica o resultado definido. A consistência varia de 0,00 a 1,00. 
Proteção contra Choques Elétricos não obtiveram combinação consistente, logo, esses itens não foram contemplados na análise dos resultados.

\section{Quadro 2 - Caracterização dos canteiros e equipamentos presentes nos canteiros}

\begin{tabular}{|c|c|c|}
\hline Obra & Tipo de Certificação & Equipamentos observados \\
\hline A1 & ISO 9000 & $\begin{array}{c}\text { Andaime Simplesmente Apoiado, Serra Circular, Máquinas } \\
\text { Equipamentos e Ferramentas }\end{array}$ \\
\hline A2 & ISO 9000 & $\begin{array}{l}\text { Andaime Suspenso Mecânico, Proteção contra Choques Elétricos, } \\
\text { Escada de Mão }\end{array}$ \\
\hline A3 & ISO 9000 & $\begin{array}{c}\text { Andaime Suspenso Mecânico, Serra Circular, Máquinas } \\
\text { Equipamentos e Ferramentas, Proteção contra Choques Elétricos, } \\
\text { Escada de Mão, Plataforma de Proteção }\end{array}$ \\
\hline A4 & ISO 9000 & Plataforma de Proteção \\
\hline B1 & $\begin{array}{c}\text { ISO 9000, ISO } 14001 \\
\text { OHSAS } 18001\end{array}$ & $\begin{array}{c}\text { Andaime Suspenso Mecânico, Máquinas Equipamentos e } \\
\text { Ferramentas, Proteção contra Choques Elétricos, Plataforma de } \\
\text { Proteção }\end{array}$ \\
\hline B2 & $\begin{array}{l}\text { ISO } 9000, \text { ISO } 14001 \\
\text { OHSAS } 18001\end{array}$ & $\begin{array}{c}\text { Andaime Simplesmente Apoiado, Andaime Fachadeiros, Máquinas } \\
\text { Equipamentos e Ferramentas, Escada de Mão }\end{array}$ \\
\hline B3 & $\begin{array}{l}\text { ISO 9000, ISO 14001, } \\
\text { OHSAS } 18001\end{array}$ & Andaime Fachadeiros \\
\hline C1 & ISO 9000 & $\begin{array}{c}\text { Andaime Simplesmente Apoiado, Andaime Fachadeiros, Máquinas } \\
\text { Equipamentos e Ferramentas, Proteção contra Choques Elétricos, } \\
\text { Escada de Mão }\end{array}$ \\
\hline D1 & $\begin{array}{l}\text { ISO } 9000, \text { ISO } 14001 \\
\text { OHSAS } 18001\end{array}$ & $\begin{array}{c}\text { Andaime Fachadeiros, Máquinas Equipamentos e Ferramentas, } \\
\text { Proteção contra Choques Elétricos, Escada Mão }\end{array}$ \\
\hline E1 & ISO 9000 & $\begin{array}{c}\text { Serra Circular, Máquinas Equipamentos e Ferramentas, Proteção } \\
\text { contra Choques Elétricos, Escada de Mão }\end{array}$ \\
\hline $\mathbf{E 2}$ & ISO 9000 & Andaime Suspenso Mecânico \\
\hline
\end{tabular}

Fonte: Os Autores

Os dados referentes aos Andaimes Fachadeiros lançados no $f_{s} / Q C A$ são os constantes na Tabela 1, a seguir. A primeira coluna refere-se ao canteiro que possuía o equipamento; a três seguintes, ao uso da certificação, neste quesito, para caracterizar a existência da norma se adotou duas condições, onde, 1 indica a existência e 0 indica a inexistência. A coluna 'Média Ponderada' refere-se ao resultado esperado obtido da média ponderada do item em análise. A coluna 'Média Ponderada Calibrada' refere-se aos valores da 'Média Ponderada' calibrado, porque, segundo Ragin (2009), é necessário fazer a calibração dos dados, pois o fs/QCA permite apenas conjuntos no intervalo entre 0 e 1 . Após processar os dados no software é obtido os dados constantes na Tabela 2.

Tabela 1 - Dados lançados no fs/QCA

\begin{tabular}{c|c|c|c|c|c}
\hline \multirow{2}{*}{ Obra } & \multicolumn{3}{|c|}{ Certificação } & $\begin{array}{c}\text { Média } \\
\text { Ponderada }\end{array}$ & $\begin{array}{c}\text { Média Ponderada } \\
\text { Calibrada }\end{array}$ \\
\cline { 2 - 6 } & Qualidade & Ambiental & Segurança & 1,63 & 1,00 \\
\hline B2 & 1 & 1 & 1 & 2,50 & 1,00 \\
\hline B3 & 1 & 1 & 0 & 1,21 & 0,10 \\
\hline C1 & 1 & 0 & 1 & 2,10 & 0,97 \\
\hline D1 & 1 & 1 & & & \\
\hline
\end{tabular}


A Tabela Verdade para os Andaimes Fachadeiros está apresentada na Tabela 2. Cada linha da Tabela Verdade indica uma combinação de certificação. A coluna frequência demonstra a quantidade de canteiros que apresentaram as combinações em cada linha e a consistência relacionada ao desempenho de segurança com as combinações de certificações, de modo a indicar aquelas que obtiveram maior relevância. Já a coluna DS (Desempenho de Segurança) só é preenchida após a análise da frequência e da consistência para gerar a solução final. Observa-se que neste item, somente uma combinação obteve consistência acima de 0,80 .

Tabela 2 - Tabela Verdade: Andaimes Fachadeiros

\begin{tabular}{c|c|c|c|c|c}
\hline Qualidade & Ambiental & Segurança & Frequência & DS & Consistência \\
\hline 1 & 0 & 0 & 3 & 1 & 0.990000 \\
\hline 1 & 1 & 1 & 1 & 0 & 0.100000 \\
\hline 1 & 1 & 0 & 0 & 0 & 0.000000 \\
\hline 1 & 0 & 1 & 0 & 0 & 0.000000 \\
\hline 0 & 1 & 1 & 0 & 0 & 0.000000 \\
\hline 0 & 1 & 0 & 0 & 0 & 0.000000 \\
\hline 0 & 0 & 1 & 0 & 0 & 0.000000 \\
\hline 0 & 0 & 0 & 0 & 0 & 0.000000 \\
\hline
\end{tabular}

O software gera três tipos de soluções: a complexa, a parcial e a intermediaria Na primeira, os 'restos' não são considerados na solução; Na segunda, qualquer 'resto' que vai ajudar a gerar uma solução logicamente mais simples é usado, independentemente de constituir um caso hipotético "fácil" ou "difícil"; Na terceira, apenas "restos" que são "fáceis" estão autorizados a ser incorporados na solução. Neste caso, os "restos" são vistos como combinações lógicas que não foram observadas nos casos (RAGIN, 2009). Na análise dos dados foi avaliada apenas a solução complexa, pois, observou-se que as combinações finais apareciam tanto na solução complexa, quanto na intermediária.

Após a análise, são obtidos quatro resultados presentes no Quadro 3. Na primeira coluna tem-se a combinação causal para obter um melhor desempenho no item Andaimes Fachadeiros. A segunda coluna indica a proporção da presença do resultado explicado por cada termo da solução. Enquanto que a terceira coluna indica a proporção de presença no resultado explicado separadamente para cada termo da solução. Por fím, a última coluna representa o grau em que as combinações têm consistência com o resultado.

\section{Quadro 3 - Desempenho de Segurança Andaime Fachadeiros}

\begin{tabular}{|l|l|l|l|}
\hline Combinação Causal & Cobertura Crua & Cobertura Exclusiva & Consistência \\
\hline Qualidade E Ambiental E Segurança & 0.967427 & 0.967427 & 0.990000 \\
\hline $\begin{array}{l}\text { Solução de cobertura': } 0.967427 \\
\text { Solução de Consistência: } 0.990000\end{array}$ & \\
\hline
\end{tabular}

$\mathrm{Na}$ análise dos Andaimes Fachadeiros, conforme o quadro acima, o modelo é explicado em $99 \%$, observou-se também que em $96 \%$ dos canteiros possuía a adoção

\footnotetext{
${ }^{2}$ Mede quanto o resultado é coberta (ou explicadas) por cada termo de solução e pôr a solução como um todo.
} 
das três certificações, demonstrando que o desempenho dos andaimes Fachadeiros é influenciado pelas certificações.

O processo de análise foi repetido para os demais itens avaliados e apresentado a partir do Quadro 4 até o Quadro 7. Na análise dos Andaimes Simplesmente Mecânico observou-se apenas uma combinação (Quadro 4). Observa-se que 30\% dos canteiros que utilizava o andaime simplesmente mecânico possuía as três certificações.

Quadro 4 - Desempenho de Segurança Andaime Simplesmente Mecânico

\begin{tabular}{|l|l|l|l|}
\hline Combinação Causal & Cobertura Crua & Cobertura Exclusiva & Consistência \\
\hline Qualidade E Ambiental E Segurança & 0.300310 & 0.300310 & 0.970000 \\
\hline $\begin{array}{l}\text { Solução de cobertura: 0.300310 } \\
\text { Solução de Consistência: } 0.970000\end{array}$ & \\
\hline
\end{tabular}

Quanto ao item Plataforma de Proteção (Quadro 5), utilizado em 48\% dos canteiros foi observado apenas uma combinação causal, com $97 \%$ de consistência.

Quadro 5 - Desempenho de Segurança Plataforma de Proteção

\begin{tabular}{|l|l|l|l|}
\hline Combinação Causal & Cobertura Crua & Cobertura Exclusiva & Consistência \\
\hline Qualidade E Ambiental E Segurança & 0.482587 & 0.482587 & 0.970000 \\
\hline $\begin{array}{l}\text { Solução de cobertura: 0.482587 } \\
\text { Solução de Consistência: 0.970000 }\end{array}$ & \\
\hline
\end{tabular}

Para o item Serra Circular (Quadro 6), presente em apenas 100\% dos canteiros, neste modelo, para se ter um bom desempenho é necessário apenas a presença da certificação da qualidade. Este modelo é explicado em 81,67\%.

\section{Quadro 6 - Desempenho de Segurança Serra Circular}

\begin{tabular}{|l|l|l|l|}
\hline Combinação Causal & Cobertura Crua & Cobertura Exclusiva & Consistência \\
\hline $\begin{array}{l}\text { Qualidade E NÃO Ambiental E } \\
\text { NÃO Segurança }\end{array}$ & 1.000000 & 1.000000 & 0.816667 \\
\hline $\begin{array}{l}\text { Solução de cobertura: } 1.000000 \\
\text { Solução de Consistência: } 0.816667\end{array}$ & & \\
\hline
\end{tabular}

Para o item Máquinas, Equipamentos e Ferramentas (Quadro 7), indica que em $79,3 \%$ dos canteiros foi necessário a presença da Qualidade para obter um bom desempenho. O modelo foi explicado em $82,5 \%$.

\section{Quadro 7 - Desempenho de Segurança Máquinas Equipamentos e Ferramentas}

\begin{tabular}{|l|l|l|l|}
\hline Combinação Causal & Cobertura Crua & Cobertura Exclusiva & Consistência \\
\hline $\begin{array}{l}\text { Qualidade E NÃO Ambiental E } \\
\text { NÃO Segurança }\end{array}$ & 0.793269 & 0.793269 & 0.825000 \\
\hline $\begin{array}{l}\text { Solução de cobertura: } 0.793269 \\
\text { Solução de Consistência: 0.825000 }\end{array}$ & \\
\hline
\end{tabular}

O Quadro 8 apresenta a relação entre equipamentos e soluções encontradas. Conforme a consistência, os equipamentos que requer atenção são, a Serra Circular e Máquinas Equipamentos e Ferramentas. Ainda para estes equipamentos, observou-se que a certificação de qualidade é suficiente para se ter um bom desempenho na 
segurança, enquanto as demais certificações são necessárias. Este resultado, confirma as conclusões de França (2009) quando afirma que, se uma construtora já tem implementado a NBR/ISO 9001:2000, consegue abranger suas atividades de comunicação e integrar os itens da NBR/ISO 14001:2004 e OHSAS 18001:1999.

\section{Quadro 8 - Relação Equipamentos x Soluções Encontradas}

\begin{tabular}{|l|l|c|c|}
\hline \multicolumn{1}{|c|}{ Equipamentos } & \multicolumn{1}{|c|}{ Soluções Encontradas } & Cobertura & Consistência \\
\hline Andaimes Fachadeiros & Qualidade E Ambiental E Segurança & 0.967427 & 0.990000 \\
\hline $\begin{array}{l}\text { Andaimes } \\
\text { Simplesmente Mecânico }\end{array}$ & Qualidade E Ambiental E Segurança & 0.300310 & 0.970000 \\
\hline Plataforma de Proteção & Qualidade E Ambiental E Segurança & 0.482587 & 0.970000 \\
\hline Serra Circular & Qualidade E NãO Ambiental E NÃO Segurança & 1.000000 & 0.816667 \\
\hline $\begin{array}{l}\text { Máquinas Equipamentos } \\
\text { e Ferramentas }\end{array}$ & Qualidade E NÃO Ambiental E NÃO Segurança & 0.793269 & 0.825000 \\
\hline
\end{tabular}

Todas as combinações podem ser consideradas como modelo, pois estão próximas de 1 indicando forte associação entre os fatores causais. Mas, com base no quadro observa-se que uma combinação obtida para andaimes Fachadeiros, simplesmente mecânico e plataforma de proteção, demonstrou ser mais consistente para melhor desempenho de segurança, essa combinação sugere que a declaração da existência das 3 certificações no canteiro potencializa o desempenho em segurança dos itens de componentes provisórios com montagem no canteiro, enquanto que para os equipamentos alugados ou adquiridos, o maior impacto no desempenho da segurança está relacionada a Certificação de Qualidade.

\section{CONSIDERAÇÕES FINAIS}

$\mathrm{O}$ artigo propõe o uso do $f s / Q C A$ como método que sugere relações lógicas entre diferentes fatores em situações complexas, e mostrou ser capaz de lidar com as características dos dados, pois é aplicável a pequenas amostras e inclui, de forma determinante a interação entre variáveis fuzzy, combinando uma avaliação qualitativa e quantitativa.

O modelo da certificação baseada nos critérios da NR 18 assume que a certificação de qualidade tem impacto positivo no desempenho da segurança. Demonstrando que alguns paradigmas da construção civil podem ser alterados devido a implementação das certificações, principalmente à de qualidade. Entretanto, vale ressaltar que este estudo foi o primeiro contato com uma técnica oriunda da Ciências Sociais e utilizada comumente nesta área. Com isso, observa-se uma conclusão limitada dos dados, sendo necessário ampliar o número amostral e também avaliar outros fatores determinantes no desempenho de segurança, como a inspeção e a auditoria. Enfim, espera-se que o texto ofereça um ponto de partida metodológico para futuras pesquisas quanto a adoção desta metodologia de análise. 


\section{AGRADECIMENTOS}

À CAPES pelo apoio recebido através das bolsas concedidas, a FINEP e as empresas por permitir as visitas técnicas.

\section{REFERÊNCIAS}

ALMEIDA, M.L.; SILVA, J.J.R.; RAMOS, R.E.B.; MENEZES, J.R.; VASCONCELOS, F.D.L. Interface entre sistemas de gestão como uma vantagem competitiva sustentável na construção civil. In: Encontro Nacional de Tecnologia do Ambiente Construído, XI. 2006, Florianópolis/SC. Anais... Porto Alegre: ANTAC, 2006.

BRASIL. Ministério do Trabalho e emprego. NR-18 - Condições e Meio Ambiente do Trabalho da Indústria da Construção. Redação dada pela Portaria $n^{\circ} 4$, Brasília, 1995.

BRASIL. Ministério do Trabalho e emprego. NR-28 - Fiscalização e Penalidades. Redação dada pela Portaria no 4, Brasília, 1995.

FLINN, R.; MEARNS, K.; O'CONNOR; BRYDEN, P, R. Measuring safety climate: identifying the common features. Safety Science 34 177-192, 2000.

FRANÇA, N. P. Sistema Integrado de Gestão - Qualidade, Meio Ambiente, Segurança e Saúde: recomendações para implementação em empresas construtoras de edifícios. Dissertação de Mestrado (Engenharia Civil) Universidade Estadual de Campinas. Campinas, 2009.

GLENDON, A. I; STANTON, N. A. Perspectives on Safety culture. Safety Science 34 193-214, 2000.

MENDEL, J. M.; KORJANI, M. Charles Ragin's Fuzzy Set Qualitative Comparative Analysis (fsQCA) used for linguistic summarizations. Information Sciences 202 (2012) 1-23.

RAGIN, C. C. The Comparative Method: moving beyond Qualitative and Quantitative Strategies. Berkeley: University of California Press, 1987.

Chapter 5: Qualitative Comparative Analysis Using Fuzzy Sets (fsQCA). In: RIHOUX, B.; RAGIN, C.C. (Eds.) Configurational Comparative Methods: Qualitative Comparative Analysis (QCA) and Related Techniques, SAGE, Los Angeles, CA, 2009. 